\title{
Related Suzuki-type fixed point theorems in ordered metric space
}

\author{
Outass Rida ${ }^{1}$, Chaira Karim² ${ }^{2}$ and Marhrani El Miloudi ${ }^{*}$ (10
}

${ }^{*}$ Correspondence:

marhrani@gmail.com

1 Laboratory of Algebra, Analysis and

Applications (L3A), Faculty of

Sciences Ben M'Sik, Hassan II

University of Casablanca,

Casablanca, Morocco

Full list of author information is

available at the end of the article

\begin{abstract}
In this paper, we use Suzuki-type contraction to prove three fixed point theorems for generalized contractions in an ordered space equipped with two metrics; we obtain some generalizations of the Kannan fixed point theorem. Our results on partially ordered metric spaces generalize and extend some results of Ran and Reurings as well as of Nieto and Rodríguez-López. To illustrate the effectiveness of our main result, we give an application to matrix equations which involves monotone mappings.
\end{abstract}

MSC: Primary $47 \mathrm{H} 10$; secondary $54 \mathrm{H} 25$

Keywords: Generalized Kannan mappings; Fixed point theorem; Suzuki-type contraction; Partially ordered metric spaces

\section{Introduction}

It is well known that the Banach fixed point theorem plays a very important part in the resolution of various problems in nonlinear analysis such as integral equations and various nonlinear problems. It also has applications in various scientific disciplines. This theorem knew intense generalizations by the introduction of various type of contractions. We cite for example the generalizations obtained by Suzuki [20], Kikkawa and Suzuki [10], Mot and Petrusel [15], Dorić and Lazović [6], Bose and Roychowdhury [2], Singh, and Swami, Mishra, Chugh and Kamal [19].

\section{Methods}

Let $(X, d)$ be a metric space and $T$ be a self-mapping on $X$.

$T$ is called a generalized Kannan mapping if there exists $r \in\left[0, \frac{1}{2}[\right.$ such that

$$
d(T x, T y) \leq r \max \{d(x, T x), d(y, T y)\}, \quad \text { for all } x, y \in X
$$

$T$ is called a Chatterjea mapping if there exists $r \in\left[0, \frac{1}{2}[\right.$ such that

$$
d(T x, T y) \leq r \max \{d(x, T y), d(y, T x)\}, \quad \text { for all } x, y \in X
$$

Kannan [8] (resp. Chatterjea [5]) shows the following results: If $(X, d)$ is a complete metric space then every generalized Kannan (resp. Chatterjea) mapping) has a unique fixed point.

(c) The Author(s) 2020. This article is licensed under a Creative Commons Attribution 4.0 International License, which permits use, sharing, adaptation, distribution and reproduction in any medium or format, as long as you give appropriate credit to the original author(s) and the source, provide a link to the Creative Commons licence, and indicate if changes were made. The images or other third party material in this article are included in the article's Creative Commons licence, unless indicated otherwise in a credit line to the material. If material is not included in the article's Creative Commons licence and your intended use is not permitted by statutory regulation or exceeds the permitted use, you will need to obtain permission directly from the copyright holder. To view a copy of this licence, visit http://creativecommons.org/licenses/by/4.0/. 
Theorem 2.1 (Kikkawa and Suzuki [10]) Let T be a mapping on a complete metric space $(X, d)$ and $\phi$ be a non-increasing function from $\left[0,1[\right.$ onto $\left.] \frac{1}{2}, 1\right]$ defined by

$$
\phi(r)= \begin{cases}1 & \text { if } 0 \leq r<\frac{\sqrt{5}-1}{2} \\ \frac{1-r}{r^{2}} & \text { if } \frac{\sqrt{5}-1}{2} \leq r<\frac{1}{\sqrt{2}} \\ \frac{1}{1+r} & \text { if } \frac{1}{\sqrt{2}} \leq r<1\end{cases}
$$

Assume that there exists $r \in[0,1[$ such that, for all $x, y \in X$,

$$
\phi(r) d(x, T x) \leq d(x, y) \quad \text { implies } \quad d(T x, T y) \leq r \max \{d(x, T x), d(y, T y)\} .
$$

Then $T$ has a unique fixed point $x^{*}$ and $\lim _{n \rightarrow+\infty} T^{n} x=x^{*}$ holds for every $x \in X$.

In 1981, Fisher presented some related fixed points theorems involving two mappings on metrics spaces under some conditions on contractions.

Theorem 2.2 (Fischer [7]) Let $(X, d)$ and $(Y, \delta)$ be two metric spaces such that $(X, d)$ is complete. Let $T: X \rightarrow Y$ and $S: Y \rightarrow X$ be two mappings such that, for all $(x, y) \in X \times Y$,

$$
\left\{\begin{array}{l}
d(S y, S T x) \leq c \cdot \max \{d(x, S y), \delta(y, T x), d(x, S T x)\} \\
\delta(T x, T S y) \leq c \cdot \max \{d(x, S y), \delta(y, T x), \delta(y, T S y)\}
\end{array}\right.
$$

where $c \in\left[0,1\left[\right.\right.$. Then there exists a unique pair $\left(x^{*}, y^{*}\right) \in X \times Y$ such that $T x^{*}=y^{*}$ and $S y^{*}=x^{*}$. Consequently, $S T x^{*}=x^{*}$ and $T S y^{*}=y^{*}$.

Some generalizations of this result have appeared in many different directions. For details, we refer to $[1,3,4,8]$ and [16].

Chaira and Marhrani proved the following result which generalized the theorem of Fischer.

Theorem $2.3([4])$ Let $(X, d)$ and $(Y, \delta)$ be two metric spaces such that $(X, d)$ is complete; let $T: X \rightarrow Y$ and $S: Y \rightarrow X$ be two mappings satisfying the following condition:

For all $x, y \in X$, one of the conditions

1. $d(x, S T x) \leq d(x, S y)$,

2. $\delta(y, T S y) \leq \delta(y, T x)$,

implies

$$
\left\{\begin{array}{l}
\delta(T x, T S y) \leq \alpha(\delta(x, S y)) \cdot \max \{\delta(y, T S y), d(x, S T x), \delta(x, S y)\} \\
d(S y, S T x) \leq \alpha(d(y, T x)) \cdot \max \{\delta(y, T S y), d(x, S T x), d(y, T x)\}
\end{array}\right.
$$

where $\alpha:\left[0,+\infty\left[\rightarrow\left[0,1\left[\right.\right.\right.\right.$ satisfies $\lim \sup _{t \rightarrow t_{0}^{+}} \alpha(t)<1$, for all $t_{0} \in[0,+\infty[$. Then there exists a unique pair $\left(x^{*}, y^{*}\right) \in X \times Y$ such that $T x^{*}=y^{*}$ and $S y^{*}=x^{*}$. And consequently, $S T x^{*}=x^{*}$ and $T S y^{*}=y^{*}$. 
In this article, we give a generalization of these results in the framework of ordered space equipped with two metrics by using the function $\psi_{r_{0}}:[0,1[\rightarrow] 0,1]$ defined by

$$
\psi_{r_{0}}(r)= \begin{cases}1 & \text { if } 0 \leq r<r_{0} \\ 1-r & \text { if } r_{0} \leq r<1\end{cases}
$$

for any $\left.r_{0} \in\right] 0,1$. Our results on partially ordered metric spaces generalize the Ran and Reurings ([17]), Nieto and Rodríguez-López ([13]) fixed point theorems.

We also give an application to matrix equations to illustrate our results.

Let us recall some basic notions which will be used in our main discussions.

Definition 2.4 Let $X$ be a nonempty set. Then $(X, d, \preceq)$ is called an ordered metric space if:

(i) $(X, d)$ is a metric space,

(ii) $(X, \preceq)$ is a partially ordered set.

Definition 2.5 ([18]) Let $(X, \preceq)$ be a partially ordered set. We say that:

(i) $x, y \in X$ are comparable if $x \leq y$ or $y \preceq x$ holds.

(ii) $f: X \longrightarrow X$ is monotone nondecreasing if $x, y \in X, x \preceq y \Longrightarrow f(x) \preceq f(y)$.

Definition 2.6 It is said that a partially ordered metric space $X$ verifies the property (P) if

(i) for any nondecreasing sequence $\left(x_{n}\right)_{n} \subset X$ converging to $x \in X$, we have $x_{n} \preceq x$ for all $n$;

(ii) for any sequence $\left(x_{n}\right)_{n} \subset X$ which converges to $x$ and $y \in X$ such that $x_{n} \preceq y$ for all integer $\mathrm{n}$, we have $x \preceq y$.

\section{Main results}

Theorem 3.1 Let $(X, \preceq)$ be a partially ordered set endowed with two metrics $d$ and $\delta$ such that $(X, d)$ is complete satisfying the property $(P)$ and $\left.\left.r_{0} \in\right] 0, \frac{-1+\sqrt{5}}{2}\right]$. Let $T$ be a monotone nondecreasing mapping on $X$. If there exists $r \in[0,1[$ such that, for all $x, y \in X$ comparable, one of the conditions

(i) $\psi_{r_{0}}(r) d(x, T x) \leq d(x, y)$,

(ii) $\psi_{r_{0}}(r) \delta(x, T x) \leq \delta(x, y)$, implies

$$
\left\{\begin{array}{l}
d(T x, T y) \leq r \max \{\delta(x, T x), d(y, T y)\}, \\
\delta(T x, T y) \leq r \max \{\delta(y, T y), d(x, T x)\},
\end{array}\right.
$$

and if there exists $x_{0} \in X$ such that $x_{0} \preceq T x_{0}$, then there exists $x^{*} \in X$ such that $T x^{*}=x^{*}$ and

$$
\lim _{n \rightarrow+\infty} d\left(T^{n} x_{0}, x^{*}\right)=\lim _{n \rightarrow+\infty} \delta\left(T^{n} x_{0}, x^{*}\right)=0 .
$$

Moreover, the fixed point is unique if the set of fixed points $\mathcal{F}(T)$ is totally ordered. 
Proof If $T\left(x_{0}\right)=x_{0}$ then the proof is finished. Assume that $T\left(x_{0}\right) \neq x_{0}$.

Since $x_{0} \prec T\left(x_{0}\right)$ and $T$ is monotone nondecreasing, we obtain by induction that

$$
x_{0} \prec T\left(x_{0}\right) \preceq T^{2}\left(x_{0}\right) \preceq \cdots \preceq T^{n}\left(x_{0}\right) \preceq T^{n+1}\left(x_{0}\right) \preceq \cdots
$$

Put $x_{n}=T^{n}\left(x_{0}\right)$ for all $n \geq 0$. Since $x_{n} \preceq x_{n+1}$ for all $n \in \mathbb{N}$, and for any integer $n$, we have $\psi_{r_{0}}(r) d\left(x_{n}, T x_{n}\right) \leq d\left(x_{n}, x_{n+1}\right)$, then

$$
\left\{\begin{array}{l}
d\left(T x_{n}, T x_{n+1}\right) \leq r \max \left\{\delta\left(x_{n}, T x_{n}\right), d\left(x_{n+1}, T x_{n+1}\right)\right\} \\
\delta\left(T x_{n}, T x_{n+1}\right) \leq r \max \left\{d\left(x_{n}, T x_{n}\right), \delta\left(x_{n+1}, T x_{n+1}\right)\right\}
\end{array}\right.
$$

hence

$$
\left\{\begin{array}{l}
d\left(T x_{n}, T x_{n+1}\right) \leq r \delta\left(x_{n}, x_{n+1}\right), \\
\delta\left(T x_{n}, T x_{n+1}\right) \leq r d\left(x_{n}, x_{n+1}\right),
\end{array}\right.
$$

which implies

$$
\begin{aligned}
& \left\{\begin{array}{l}
d\left(x_{n+1}, x_{n+2}\right) \leq r \max \left\{d\left(x_{n}, x_{n+1}\right), \delta\left(x_{n}, x_{n+1}\right)\right\}, \\
\delta\left(x_{n+1}, x_{n+2}\right) \leq r \max \left\{d\left(x_{n}, x_{n+1}\right), \delta\left(x_{n}, x_{n+1}\right)\right\},
\end{array}\right. \\
& \left\{\begin{array}{l}
d\left(x_{n+1}, x_{n+2}\right) \leq r^{n+1} \max \left\{d\left(x_{o}, x_{1}\right), \delta\left(x_{o}, x_{1}\right)\right\}, \\
\delta\left(x_{n+1}, x_{n+2}\right) \leq r^{n+1} \max \left\{\delta\left(x_{o}, x_{1}\right), d\left(x_{o}, x_{1}\right)\right\} .
\end{array}\right.
\end{aligned}
$$

Consequently, $\left(x_{n}\right)_{n \geq 0}$ is a Cauchy sequence in $(X, d)$ and $(X, \delta)$. Then there exists an element $x^{*}$ in $X$, such that $\lim _{n \rightarrow+\infty} d\left(x_{n}, x^{*}\right)=0$.

Since $X$ satisfies the property $(\mathrm{P})$ and $x_{n} \rightarrow x^{*}$, then $x_{n} \preceq x^{*}$ for all $n \geq 0$. As $T$ is a monotone nondecreasing, we obtain

$$
x_{n} \preceq T x^{*}, \quad \text { for all } \mathrm{n} \text {, }
$$

and by our assumption $x^{*} \preceq T x^{*}$.

We assert that $T x^{*}=x^{*}$. Assume that this is not the case.

If there exists a nondecreasing function $\phi: \mathbb{N} \longrightarrow \mathbb{N}$ such that

$$
d\left(x_{\phi(n)}, T x_{\phi(n)}\right)>d\left(x_{\phi(n)}, T x^{*}\right)
$$

and since $\lim _{n} d\left(x_{\phi(n)}, T x_{\phi(n)}\right)=0$, we obtain $\lim _{n} d\left(x_{\phi(n)}, T x^{*}\right)=0$; this contradicts our hypothesis. Then

$$
d\left(x_{n}, T x_{n}\right) \leq d\left(x_{n}, T x^{*}\right), \quad \text { for large integers } n,
$$

First case: $r \in\left[0, r_{0}\left[\right.\right.$. Since $x_{n} \preceq T x^{*}$ for all $\mathrm{n}$, we have

$$
\left\{\begin{array}{l}
d\left(x_{n+1}, T^{2} x^{*}\right) \leq r \max \left\{\delta\left(x_{n}, x_{n+1}\right), d\left(T x^{*}, T^{2} x^{*}\right)\right\} \\
\delta\left(x_{n+1}, T^{2} x^{*}\right) \leq r \max \left\{d\left(x_{n}, x_{n+1}\right), \delta\left(T x^{*}, T^{2} x^{*}\right)\right\}
\end{array}\right.
$$


and then

$$
d\left(x^{*}, T^{2} x^{*}\right) \leq r d\left(T x^{*}, T^{2} x^{*}\right) .
$$

On the other hand $x^{*} \preceq T x^{*}$, then

$$
\begin{aligned}
& \left\{\begin{array}{l}
d\left(T x^{*}, T^{2} x^{*}\right) \leq r \max \left\{\delta\left(x^{*}, T x^{*}\right), d\left(T x^{*}, T^{2} x^{*}\right)\right\} \\
\delta\left(T x^{*}, T^{2} x^{*}\right) \leq r \max \left\{d\left(x^{*}, T x^{*}\right), \delta\left(T x^{*}, T^{2} x^{*}\right)\right\}
\end{array}\right. \\
& \left\{\begin{array}{l}
d\left(T x^{*}, T^{2} x^{*}\right) \leq r \delta\left(x^{*}, T x^{*}\right), \\
\delta\left(T x^{*}, T^{2} x^{*}\right) \leq r d\left(x^{*}, T x^{*}\right) .
\end{array}\right.
\end{aligned}
$$

Assume that

$$
d\left(T x^{*}, T^{2} x^{*}\right)>d\left(x^{*}, T x^{*}\right) \text { and } \delta\left(T x^{*}, T^{2} x^{*}\right)>\delta\left(x^{*}, T x^{*}\right) .
$$

If $\delta\left(x^{*}, T x^{*}\right) \leq d\left(x^{*}, T x^{*}\right)$. We have

$$
d\left(x^{*}, T x^{*}\right)<d\left(T^{2} x^{*}, T x^{*}\right) \leq r \delta\left(x^{*}, T x^{*}\right) \leq d\left(x^{*}, T x^{*}\right),
$$

which is absurd. The same conclusion is obtained if $d\left(x^{*}, T x^{*}\right) \leq \delta\left(x^{*}, T x^{*}\right)$. These considerations imply that one of the inequalities

$$
d\left(T x^{*}, T^{2} x^{*}\right) \leq d\left(x^{*}, T x^{*}\right) \quad \text { or } \quad \delta\left(T x^{*}, T^{2} x^{*}\right) \leq \delta\left(x^{*}, T x^{*}\right)
$$

holds. For $x=T x^{*}$ and $y=x^{*}$, we have

$$
\left\{\begin{array}{l}
d\left(T x^{*}, T^{2} x^{*}\right) \leq r \max \left\{\delta\left(T x^{*}, T^{2} x^{*}\right), d\left(x^{*}, T x^{*}\right)\right\}, \\
\delta\left(T x^{*}, T^{2} x^{*}\right) \leq r \max \left\{d\left(T x^{*}, T^{2} x^{*}\right), \delta\left(x^{*}, T x^{*}\right)\right\} .
\end{array}\right.
$$

Using (2), we obtain

$$
\left\{\begin{array}{l}
d\left(T x^{*}, T^{2} x^{*}\right) \leq r d\left(x^{*}, T x^{*}\right) \\
\delta\left(T x^{*}, T^{2} x^{*}\right) \leq r \delta\left(x^{*}, T x^{*}\right)
\end{array}\right.
$$

We deduce from these relations that

$$
\left.d\left(x^{*}, T x^{*}\right) \leq d\left(x^{*}, T^{2} x^{*}\right)+d\left(T^{2} x^{*}, T x^{*}\right)\right\} \leq\left(r^{2}+r\right) d\left(x^{*}, T x^{*}\right) .
$$

Since $r^{2}+r<1$, we conclude to a contradiction with our hypothesis $T x^{*} \neq x^{*}$.

Second case: $r \in\left[r_{0}, 1\left[\right.\right.$. Put $A=\left\{z \in X ; z\right.$ and $x_{n}$ are comparable, $\left.\forall n \in \mathbb{N}\right\}$. $A$ is nonempty, since $x_{0}$ and $x^{*}$ belongs to $A$. 
Let $z \in A \backslash\left\{x^{*}\right\}$, we have

$$
\begin{cases}\forall n \in \mathbb{N} & x_{n} \preceq x^{*} \prec z, \\ \text { or } & \\ \forall n \in \mathbb{N} & z \preceq x_{0} \preceq x_{n}, \\ \text { or } & \\ \exists n_{0} \in \mathbb{N} & x_{n_{0}} \preceq z \prec x_{n_{0}+1} \preceq x^{*},\end{cases}
$$

and

$$
\psi_{r_{0}}(r) d\left(x_{n}, T x_{n}\right) \leq r d\left(x_{n}, z\right), \quad \text { for large integers } n,
$$

therefore

$$
d\left(x_{n+1}, T z\right) \leq r \max \left\{\delta\left(x_{n}, x_{n+1}\right), d(z, T z)\right\},
$$

which leads to

$$
d\left(x^{*}, T z\right) \leq r d(z, T z) .
$$

The inequality $d(z, T z) \leq d\left(z, x^{*}\right)+d\left(x^{*}, T z\right) \leq d\left(z, x^{*}\right)+r d(z, T z)$ implies

$$
\psi_{r_{0}}(r) d(z, T z) \leq d\left(z, x^{*}\right) .
$$

By (3), $z$ and $x^{*}$ are comparable. Then

$$
d\left(T z, T x^{*}\right) \leq r \max \left\{\delta(z, T z), d\left(x^{*}, T x^{*}\right)\right\} .
$$

If $\left(x_{n}\right)_{n}$ is a stationary sequence, we have $x^{*}=T x^{*}$ and if not, there exists a subsequence $\left(x_{\phi(n)}\right)_{n}$, such that $x_{\phi(n)} \neq x^{*}$, for all $n$. And consequently, for $z=x_{\phi(n)}$, we have

$$
d\left(T x_{\phi(n)}, T x^{*}\right) \leq r \max \left\{\delta\left(x_{\phi(n)}, T x_{\phi(n)}\right), d\left(x^{*}, T x^{*}\right)\right\},
$$

which leads to

$$
d\left(x^{*}, T x^{*}\right) \leq r d\left(x^{*}, T x^{*}\right) .
$$

These considerations permit us to conclude that $x^{*}=T x^{*}$. These conclusions contradict the fact $T x^{*} \neq x^{*}$.

And then we conclude that $T x^{*}=x^{*}$.

We have $\lim _{n \rightarrow+\infty} \delta\left(x_{n}, x^{*}\right)=0$. If not, $\left(\delta\left(x_{n}, x^{*}\right)\right)_{n}$ does not converge to 0 , then

$$
\delta\left(x_{n}, T x_{n}\right) \leq \delta\left(x_{n}, x^{*}\right), \quad \text { for large integers } n,
$$

since $x_{n} \preceq x^{*}$ for all $\mathrm{n}$, consequently

$$
\delta\left(x_{n+1}, x^{*}\right)=\delta\left(x_{n+1}, T x^{*}\right) \leq r \max \left\{d\left(x_{n}, T x_{n}\right), \delta\left(x^{*}, T x^{*}\right)\right\}=r d\left(x_{n}, T x_{n}\right) .
$$

Thus, $\lim _{n \rightarrow+\infty} \delta\left(x_{n+1}, x^{*}\right)=0$, which is a contradiction. Hence, $\lim _{n \rightarrow+\infty} \delta\left(x_{n}, x^{*}\right)=0$. 
For uniqueness: Let $z \in X$ such that $T z=z$.

We have $d(z, T z)=0 \leq d\left(z, x^{*}\right)$, since $\mathcal{F}(T)$ is totally ordered,

$$
d\left(z, x^{*}\right)=d\left(T z, T x^{*}\right) \leq r \max \left\{\delta(z, T z), d\left(x^{*}, T x^{*}\right)\right\}=0 .
$$

Example 3.2 Let $X=[0,1]^{2}$. We define a partial order “ $\preceq$ " in $\mathrm{X}$ as

$$
\left(x, x^{\prime}\right) \preceq\left(y, y^{\prime}\right) \text { if and only if } \quad x \geq y \quad \text { and } \quad x^{\prime} \geq y^{\prime} .
$$

Define $d$ and $\delta$ by

$$
d\left(\left(x, x^{\prime}\right),\left(y, y^{\prime}\right)\right)=|x-y|+\left|x^{\prime}-y^{\prime}\right| \quad \text { and } \quad \delta\left(\left(x, x^{\prime}\right),\left(y, y^{\prime}\right)\right)=\max \left(|x-y|,\left|x^{\prime}-y^{\prime}\right|\right)
$$

for all $\left(\left(x, x^{\prime}\right),\left(y, y^{\prime}\right)\right) \in X^{2}$.

Thus $(X, d, \preceq)$ and $(X, \delta, \preceq)$ are two ordered metrics spaces. And define $T: X \rightarrow X$ by

$$
T\left(x, x^{\prime}\right)=\left(\frac{x^{2}}{5}, \frac{y^{2}}{5}\right)
$$

We put $r_{0}=\frac{-1+\sqrt{5}}{2}$ and $r=\frac{1}{4}$. The map $\mathrm{T}$ is nondecreasing, and the space $X$ satisfies the property (P) for $d$ and $\delta$. For all comparable $\left(x, x^{\prime}\right),\left(y, y^{\prime}\right) \in X$ such that $\left(x, x^{\prime}\right) \preceq\left(y, y^{\prime}\right)$, we have

$$
\begin{aligned}
d\left(T\left(x, x^{\prime}\right), T\left(y, y^{\prime}\right)\right) & =d\left(\left(\frac{x^{2}}{5}, \frac{x^{\prime 2}}{5}\right),\left(\frac{y^{2}}{5}, \frac{y^{\prime 2}}{5}\right)\right) \\
& =\frac{x^{2}}{5}-\frac{y^{2}}{5}+\frac{x^{\prime 2}}{5}-\frac{y^{\prime 2}}{5} \\
& \leq \frac{x^{2}}{5}+\frac{x^{\prime 2}}{5} \\
& \leq \frac{1}{4}\left(x-\frac{x^{2}}{5}\right)+\frac{1}{4}\left(x^{\prime}-\frac{x^{\prime 2}}{5}\right) \\
& \leq \frac{1}{4} d\left(\left(x, x^{\prime}\right), T\left(x, x^{\prime}\right)\right) .
\end{aligned}
$$

Hence

$$
d\left(T\left(x, x^{\prime}\right), T\left(y, y^{\prime}\right)\right) \leq \frac{1}{4} \max \left\{d\left(\left(x, x^{\prime}\right), T\left(x, x^{\prime}\right)\right), \delta\left(\left(y, y^{\prime}\right), T\left(y, y^{\prime}\right)\right)\right\} .
$$

In the same way

$$
\delta\left(T\left(x, x^{\prime}\right), T\left(y, y^{\prime}\right)\right) \leq \frac{1}{4} \max \left\{\delta\left(\left(x, x^{\prime}\right), T\left(x, x^{\prime}\right)\right), d\left(\left(y, y^{\prime}\right), T\left(y, y^{\prime}\right)\right)\right\} .
$$

Moreover, $\left(\frac{1}{2}, \frac{1}{2}\right) \preceq T\left(\frac{1}{2}, \frac{1}{2}\right)$. Therefore, $T$ satisfies the hypotheses of Theorem 3.1. Hence, $T$ has a unique fixed point $x^{*}=(0,0)$.

If we assume, in the above theorem, that $d=\delta$ and $\alpha$ is a constant function, we obtain a generalization of a Kannan-type contraction [9]. 
Corollary 3.3 Let $(X, d, \preceq)$ be an ordered metric space such that $(X, d)$ is complete and $T$ a nondecreasing mapping on $(X, \preceq)$. Assume that there exists $r=2 \alpha$, where $\alpha \in\left[0, \frac{1}{2}[\right.$ such that, for all comparable $x, y \in X$,

$$
\psi_{r_{0}}(r) d(x, T x) \leq d(x, y) \quad \text { implies } \quad d(T x, T y) \leq \alpha . d(x, T x)+\alpha . d(y, T y)
$$

where $\left.\left.r_{0} \in\right] 0, \frac{-1+\sqrt{5}}{2}\right]$.

If there exists $x_{0} \in X$ such that $x_{0} \preceq T x_{0}$ and $X$ satisfies the property $(P)$ for $d$, then there exists an element $x^{*} \in X$ such that $T x^{*}=x^{*}$ and $\lim _{n \rightarrow+\infty} T^{n} x_{0}=x^{*}$. Moreover, the fixed point of $T$ is unique if for all any pair $\{x, y\} \subset X$ admits an upper bound or a lower bound.

Proof We remark that, for all comparable $x, y \in X$,

$$
\psi_{r_{0}}(r) d(x, T x) \leq d(x, y) \quad \Rightarrow \quad d(T x, T y) \leq r \max \{d(x, T x), d(y, T y)\} .
$$

By Theorem 3.1, we conclude that $T$ has a fixed point.

For the uniqueness, we suppose that there exist two fixed points $x, y \in X$. From the hypothesis on $x$ and $y$ there exists $z$ in $X$ such that $z \preceq x$ and $z \preceq y$.

By the monotony of $T$,

$$
T^{n}(z) \preceq T^{n}(x)=x,
$$

for all $n \geq 0$. Set $z_{n}=T^{n}(z)$, and we have

$$
d(x, T x) \leq d\left(x, z_{n}\right)
$$

for all integer $n$. Then, for all $n \geq 0$,

$$
\begin{aligned}
d\left(z_{n+1}, x\right) & \leq \alpha d(x, T x)+\alpha d\left(z_{n}, z_{n+1}\right) \\
& \leq \alpha d\left(z_{n}, x\right)+\alpha d\left(z_{n+1}, x\right) \\
& \leq \frac{\alpha}{1-\alpha} d\left(z_{n}, x\right)
\end{aligned}
$$

By induction, we show that

$$
d\left(z_{n+1}, x\right) \leq\left(\frac{\alpha}{1-\alpha}\right)^{n+1} d(z, x) .
$$

Since $0 \leq \alpha<\frac{1}{2}$, we have $0 \leq \frac{\alpha}{1-\alpha}<1$. Hence,

$$
\lim _{n \rightarrow+\infty} d\left(z_{n}, x\right)=0
$$

In the same way we prove that

$$
\lim _{n \rightarrow+\infty} d\left(z_{n}, y\right)=0 .
$$


By the triangle inequality,

$$
d(x, y) \leq d\left(x, z_{n}\right)+d\left(y, z_{n}\right), \quad \forall n \in \mathbb{N},
$$

and by (5) and (6) we conclude that $x=y$.

Note that, if $X$ is not necessarily provided with a partial order, then we find the same result as that of Theorem 3.1. with some modifications in the proof consisting basically of avoiding the use of the order.

Theorem 3.4 Let $d$ and $\delta$ be two metrics on $X$ such that $(X, d)$ is complete and $r_{0} \in$ ] $\left.0, \frac{-1+\sqrt{5}}{2}\right]$. Let $T$ be a self-mapping on $X$. If there exists $r \in[0,1[$ such that, for all $x, y \in X$, one of the conditions:

(i) $\psi_{r_{0}}(r) d(x, T x) \leq d(x, y)$,

(ii) $\psi_{r_{0}}(r) \delta(x, T x) \leq \delta(x, y)$,

implies

$$
\left\{\begin{array}{l}
d(T x, T y) \leq r \max \{\delta(x, T x), d(y, T y)\}, \\
\delta(T x, T y) \leq r \max \{\delta(y, T y), d(x, T x)\},
\end{array}\right.
$$

then there exists an unique element $x^{*} \in X$ such that $T x^{*}=x^{*}$ and

$$
\lim _{n \rightarrow+\infty} d\left(T^{n} x, x^{*}\right)=\lim _{n \rightarrow+\infty} \delta\left(T^{n} x, x^{*}\right)=0
$$

holds for every $x \in X$.

Example 3.5 Let $X=\{(0,0),(4,0),(0,4),(5,0),(4,5),(5,4)\}$ and define $T$ by

$$
T\left(x, x^{\prime}\right)= \begin{cases}\left(x^{\prime}, 0\right) & \text { if } x \leq x^{\prime} \text { and }\left(x, x^{\prime}\right) \in X \backslash\{(0,4)\} \\ \left(0, x^{\prime}\right) & \text { if } x>x^{\prime} \text { and }\left(x, x^{\prime}\right) \in X \backslash\{(0,4)\} \\ (0,0) & \text { if }\left(x, x^{\prime}\right)=(0,4)\end{cases}
$$

Define $d$ and $\delta$ by

$$
d\left(\left(x, x^{\prime}\right),\left(y, y^{\prime}\right)\right)=|x-y|+\left|x^{\prime}-y^{\prime}\right| \quad \text { and } \quad \delta\left(\left(x, x^{\prime}\right),\left(y, y^{\prime}\right)\right)=\frac{\sqrt{5}}{2}\left(|x-y|+\left|x^{\prime}-y^{\prime}\right|\right)
$$

for all $\left(\left(x, x^{\prime}\right),\left(y, y^{\prime}\right)\right) \in X^{2}$.

We put $r_{0}=\frac{-1+\sqrt{5}}{2}$ and $r=\frac{2}{\sqrt{5}}$. Let $\left(\left(x, x^{\prime}\right),\left(y, y^{\prime}\right)\right) \in X^{2}$.

First case: $\left(\left(x, x^{\prime}\right),\left(y, y^{\prime}\right)\right) \notin\{((4,5),(5,4)),((5,4),(4,5))\}$, we have

$$
\left\{\begin{array}{l}
d\left(T\left(x, x^{\prime}\right), T\left(y, y^{\prime}\right)\right) \leq r \max \left\{\delta\left(\left(x, x^{\prime}\right), T\left(x, x^{\prime}\right)\right), d\left(\left(y, y^{\prime}\right), T\left(y, y^{\prime}\right)\right)\right\} \\
\delta\left(T\left(x, x^{\prime}\right), T\left(y, y^{\prime}\right)\right) \leq r \max \left\{\delta\left(\left(y, y^{\prime}\right), T\left(y, y^{\prime}\right)\right), d\left(\left(x, x^{\prime}\right), T\left(x, x^{\prime}\right)\right)\right\} .
\end{array}\right.
$$

Second case: $\left(x, x^{\prime}\right)=(4,5)$ and $\left(y, y^{\prime}\right)=(5,4)$. We have

$$
\psi_{r_{0}}(r) d\left(\left(x, x^{\prime}\right), T\left(x, x^{\prime}\right)\right)=6\left(1-\frac{1}{\sqrt{5}}\right) \text { and } d\left(\left(x, x^{\prime}\right),\left(y, y^{\prime}\right)\right)=2 \text {, }
$$




$$
\psi_{r_{0}}(r) \delta\left(\left(x, x^{\prime}\right), T\left(x, x^{\prime}\right)\right)=3(\sqrt{5}-1) \quad \text { and } \quad \delta\left(\left(x, x^{\prime}\right),\left(y, y^{\prime}\right)\right)=\sqrt{5} .
$$

Note that

$$
\psi_{r_{0}}(r) d\left(\left(x, x^{\prime}\right), T\left(x, x^{\prime}\right)\right)>d\left(\left(x, x^{\prime}\right),\left(y, y^{\prime}\right)\right)
$$

and

$$
\psi_{r_{0}}(r) \delta\left(\left(x, x^{\prime}\right), T\left(x, x^{\prime}\right)\right)>\delta\left(\left(x, x^{\prime}\right),\left(y, y^{\prime}\right)\right) .
$$

Since $d\left(T\left(x, x^{\prime}\right), T\left(y, y^{\prime}\right)\right)=9$ and $\delta\left(T\left(x, x^{\prime}\right), T\left(y, y^{\prime}\right)\right)=\frac{9 \sqrt{5}}{2}$,

$$
\left\{\begin{array}{l}
d\left(T\left(x, x^{\prime}\right), T\left(y, y^{\prime}\right)\right)>r \max \left\{\delta\left(\left(x, x^{\prime}\right), T\left(x, x^{\prime}\right)\right), d\left(\left(y, y^{\prime}\right), T\left(y, y^{\prime}\right)\right)\right\} \\
\delta\left(T\left(x, x^{\prime}\right), T\left(y, y^{\prime}\right)\right)>r \max \left\{\delta\left(\left(y, y^{\prime}\right), T\left(y, y^{\prime}\right)\right), d\left(\left(x, x^{\prime}\right), T\left(x, x^{\prime}\right)\right)\right\}
\end{array}\right.
$$

Similarly for $\left(x, x^{\prime}\right)=(5,4)$ and $\left(y, y^{\prime}\right)=(4,5)$.

Hence, $T$ satisfies the hypotheses of Theorem 3.4 but we do not have

$$
\left\{\begin{array}{l}
d\left(T\left(x, x^{\prime}\right), T\left(y, y^{\prime}\right)\right) \leq r \max \left\{\delta\left(\left(x, x^{\prime}\right), T\left(x, x^{\prime}\right)\right), d\left(\left(y, y^{\prime}\right), T\left(y, y^{\prime}\right)\right)\right\} \\
\delta\left(T\left(x, x^{\prime}\right), T\left(y, y^{\prime}\right)\right) \leq r \max \left\{\delta\left(\left(y, y^{\prime}\right), T\left(y, y^{\prime}\right)\right), d\left(\left(x, x^{\prime}\right), T\left(x, x^{\prime}\right)\right)\right\}
\end{array}\right.
$$

for all $\left(x, x^{\prime}\right),\left(y, y^{\prime}\right) \in X$. Hence, $T$ has a unique fixed point $x^{*}=(0,0)$.

With the same arguments as in the proof of Theorem 3.1, we obtain the following.

Theorem 3.6 Let $(X, \preceq)$ be a partially ordered set endowed with two metrics $d$ and $\delta$ such that $(X, d)$ is complete and $\left.\left.r_{0} \in\right] 0,2(\sqrt{2}-1)\right]$. Let $T$ be a nondecreasing mapping; and assume that there exists $r \in[0,1$ [ such that, for all comparable $x, y \in X$, one of the conditions:

(i) $\psi_{r_{0}}(r) d(x, T x) \leq d(x, y)$,

(ii) $\psi_{r_{0}}(r) \delta(x, T x) \leq \delta(x, y)$,

implies

$$
\left\{\begin{array}{l}
d(T x, T y) \leq r \max \left\{\frac{d(x, T y)+\delta(y, T x)}{2}, d(x, T x)\right\} \\
\delta(T x, T y) \leq r \max \left\{\frac{\delta(x, T y)+d(y, T x)}{2}, \delta(x, T x)\right\} .
\end{array}\right.
$$

If there exists $x_{0} \in X$ such that $x_{0} \preceq T x_{0}$ and $X$ satisfies the property $(P)$ for $d$, then there exists an element $x^{*} \in X$ such that $T x^{*}=x^{*}$ and $\lim _{n \rightarrow+\infty} d\left(T^{n} x_{0}, x^{*}\right)=\lim _{n \rightarrow+\infty} \delta\left(T^{n} x_{0}\right.$, $\left.x^{*}\right)=0$.

Proof First step. If $T\left(x_{0}\right)=x_{0}$, then the existence of a fixed point is proved. Suppose that $T\left(x_{0}\right) \neq x_{0}$. Following the lines of the proof of Theorem 2.3 we find that $\left\{x_{n}\right\}=\left\{T^{n}\left(x_{0}\right)\right\}$ is a convergent sequence in $X$. Indeed, by our assumption $x_{n}$ and $x_{n+1}$ are comparable, for every $n=0,1,2, \ldots$, for $x=x_{n}$ and $y=x_{n+1}$, we have $\psi_{r_{0}}(r) d\left(x_{n}, T x_{n}\right) \leq d\left(x_{n}, x_{n+1}\right)$; then

$$
d\left(x_{n+1}, x_{n+2}\right) \leq r \max \left\{\frac{d\left(x_{n}, x_{n+2}\right)}{2}, d\left(x_{n}, x_{n+1}\right)\right\}
$$




$$
\begin{aligned}
& \leq r \max \left\{\frac{d\left(x_{n}, x_{n+1}\right)+d\left(x_{n+1}, x_{n+2}\right)}{2}, d\left(x_{n}, x_{n+1}\right)\right\} \\
& \leq r \max \left\{d\left(x_{n+1}, x_{n+2}\right), d\left(x_{n}, x_{n+1}\right)\right\}
\end{aligned}
$$

and

$$
\begin{aligned}
\delta\left(x_{n+1}, x_{n+2}\right) & \leq r \max \left\{\frac{\delta\left(x_{n}, x_{n+2}\right)}{2}, \delta\left(x_{n}, x_{n+1}\right)\right\} \\
& \leq r \max \left\{\frac{\delta\left(x_{n}, x_{n+1}\right)+\delta\left(x_{n+1}, x_{n+2}\right)}{2}, \delta\left(x_{n}, x_{n+1}\right)\right. \\
& \leq r \max \left\{\delta\left(x_{n+1}, x_{n+2}\right), \delta\left(x_{n}, x_{n+1}\right)\right\}
\end{aligned}
$$

We deduce that

$$
\left\{\begin{array}{l}
d\left(x_{n+1}, x_{n+2}\right) \leq r d\left(x_{n}, x_{n+1}\right) \\
\delta\left(x_{n+1}, x_{n+2}\right) \leq r \delta\left(x_{n}, x_{n+1}\right)
\end{array}\right.
$$

It follows that $\left(x_{n}\right)_{n \geq 0}$ is Cauchy sequence in $(X, d)$ and $(X, \delta)$; therefore there exists an element $x^{*}$ of $X$ such that $\lim _{n} d\left(x_{n}, x^{*}\right)=0$.

Second step. Assume that $\left(\delta\left(x_{n}, x^{*}\right)\right)_{n}$ does not converge to 0 . Since $x_{n} \preceq x^{*}$ for all $\mathrm{n}$, and

$$
\delta\left(x_{n}, T x_{n}\right) \leq \delta\left(x_{n}, x^{*}\right) \quad \text { for large integers } n,
$$

we obtain

$$
\left\{\begin{array}{l}
d\left(x_{n+1}, T x^{*}\right) \leq r \max \left\{\frac{d\left(x_{n}, T x^{*}\right)+\delta\left(x^{*}, x_{n+1}\right)}{2}, d\left(x_{n}, x_{n+1}\right)\right\}, \\
\delta\left(x_{n+1}, T x^{*}\right) \leq r \max \left\{\frac{\delta\left(x_{n}, T x^{*}\right)+d\left(x^{*}, x_{n+1}\right)}{2}, \delta\left(x_{n}, x_{n+1}\right)\right\} .
\end{array}\right.
$$

The latter inequality implies $\lim _{n} \delta\left(x_{n}, T x^{*}\right)=0$; and then $x^{*} \neq T x^{*}$.

It follows that $d\left(x_{n}, T x_{n}\right) \leq d\left(x_{n}, T x^{*}\right)$, for large integers $n$; since $x_{n} \preceq T x^{*}$ for all $\mathrm{n}$,

$$
d\left(x_{n+1}, T^{2} x^{*}\right) \leq r \max \left\{\frac{d\left(x_{n}, T^{2} x^{*}\right)+\delta\left(T x^{*}, x_{n+1}\right)}{2}, d\left(x_{n}, x_{n+1}\right)\right\}
$$

which gives $T^{2} x^{*}=x^{*}$.

Otherwise for $x=x^{*}$ and $y=T x^{*}$, we have $x \preceq y$ and $\delta\left(x^{*}, T x^{*}\right) \leq \delta\left(x^{*}, T x^{*}\right)$; which implies

$$
d\left(T x^{*}, T^{2} x^{*}\right) \leq r \max \left\{\frac{d\left(x^{*}, T^{2} x^{*}\right)}{2}, d\left(x^{*}, T x^{*}\right)\right\} .
$$

Consequently, $T x^{*}=x^{*}$; this gives us a contradiction and permits us to conclude $\lim _{n} \delta\left(x_{n}\right.$, $\left.x^{*}\right)=0$.

Third step. Assume that $x^{*} \neq T x^{*}$. Since $x_{n} \preceq T x^{*}$ and for large integers, we have

$$
\psi_{r_{0}}(r) d\left(x_{n}, T x_{n}\right) \leq d\left(x_{n}, T x_{n}\right) \leq d\left(x_{n}, T x^{*}\right)
$$


which implies

$$
\left\{\begin{array}{l}
d\left(x_{n+1}, T^{2} x^{*}\right) \leq r \max \left\{\frac{d\left(x_{n}, T^{2} x^{*}\right)+\delta\left(T x^{*}, x_{n+1}\right)}{2}, d\left(x_{n}, x_{n+1}\right)\right\}, \\
\delta\left(x_{n+1}, T^{2} x^{*}\right) \leq r \max \left\{\frac{\delta\left(x_{n}, T^{2} x^{*}\right)+d\left(T x^{*}, x_{n+1}\right)}{2}, \delta\left(x_{n}, x_{n+1}\right)\right\},
\end{array}\right.
$$

thus,

$$
\left\{\begin{array}{l}
d\left(x^{*}, T^{2} x^{*}\right) \leq r \frac{d\left(x^{*}, T^{2} x^{*}\right)+\delta\left(T x^{*}, x^{*}\right)}{2}, \\
\delta\left(x^{*}, T^{2} x^{*}\right) \leq r \frac{\delta\left(x^{*}, T^{2} x^{*}\right)+d\left(T x^{*}, x^{*}\right)}{2},
\end{array}\right.
$$

and then

$$
\begin{aligned}
d\left(x^{*}, T^{2} x^{*}\right) & \leq \frac{r}{2-r} \delta\left(T x^{*}, x^{*}\right), \\
\delta\left(x^{*}, T^{2} x^{*}\right) & \leq \frac{r}{2-r} d\left(T x^{*}, x^{*}\right) .
\end{aligned}
$$

For $x=x^{*}$ and $y=T x^{*}$, we have

$$
\begin{aligned}
d\left(T x^{*}, T^{2} x^{*}\right) & \leq r \max \left\{\frac{d\left(x^{*}, T^{2} x^{*}\right)}{2}, d\left(x^{*}, T x^{*}\right)\right\} \\
& \leq r \max \left\{\frac{r}{2(2-r)} \delta\left(x^{*}, T x^{*}\right), d\left(x^{*}, T x^{*}\right)\right\}
\end{aligned}
$$

and

$$
\begin{aligned}
\delta\left(T x^{*}, T^{2} x^{*}\right) & \leq r \max \left\{\frac{\delta\left(x^{*}, T^{2} x^{*}\right)}{2}, \delta\left(x^{*}, T x^{*}\right)\right\} \\
& \leq r \max \left\{\frac{r}{2(2-r)} d\left(x^{*}, T x^{*}\right), \delta\left(x^{*}, T x^{*}\right)\right\}
\end{aligned}
$$

Assume that

$$
d\left(T x^{*}, T^{2} x^{*}\right)>d\left(x^{*}, T x^{*}\right) \text { and } \delta\left(T x^{*}, T^{2} x^{*}\right)>\delta\left(x^{*}, T x^{*}\right) .
$$

If $d\left(x^{*}, T x^{*}\right) \leq \delta\left(x^{*}, T x^{*}\right)$, the inequality (9) implies

$$
\delta\left(T x^{*}, T^{2} x^{*}\right) \leq \delta\left(x^{*}, T x^{*}\right)<\delta\left(T x^{*}, T^{2} x^{*}\right)
$$

and if $d\left(x^{*}, T x^{*}\right) \geq \delta\left(x^{*}, T x^{*}\right)$, the inequality (8) implies

$$
d\left(T x^{*}, T^{2} x^{*}\right)<d\left(T x^{*}, T^{2} x^{*}\right) .
$$

These considerations permit us to conclude that one of the inequalities

$$
d\left(T x^{*}, T^{2} x^{*}\right) \leq d\left(x^{*}, T x^{*}\right) \quad \text { or } \quad \delta\left(T x^{*}, T^{2} x^{*}\right) \leq \delta\left(x^{*}, T x^{*}\right)
$$


is satisfied. And consequently

$$
\left\{\begin{array}{l}
d\left(T x^{*}, T^{2} x^{*}\right) \leq r \max \left\{\frac{\delta\left(x^{*}, T^{2} x^{*}\right)}{2}, d\left(T x^{*}, T^{2} x^{*}\right)\right\}, \\
\delta\left(T x^{*}, T^{2} x^{*}\right) \leq r \max \left\{\frac{d\left(x^{*}, T^{2} x^{*}\right)}{2}, \delta\left(T x^{*}, T^{2} x^{*}\right)\right\}
\end{array}\right.
$$

so

$$
\left\{\begin{array}{l}
d\left(T x^{*}, T^{2} x^{*}\right) \leq \frac{r}{2} \delta\left(x^{*}, T^{2} x^{*}\right) \\
\delta\left(T x^{*}, T^{2} x^{*}\right) \leq \frac{r}{2} d\left(x^{*}, T^{2} x^{*}\right)
\end{array}\right.
$$

Therefore by (7)

$$
\left\{\begin{array}{l}
d\left(T x^{*}, T^{2} x^{*}\right) \leq \frac{r^{2}}{2(2-r)} d\left(x^{*}, T x^{*}\right), \\
\delta\left(T x^{*}, T^{2} x^{*}\right) \leq \frac{r^{2}}{2(2-r)} \delta\left(x^{*}, T x^{*}\right) .
\end{array}\right.
$$

It follows that

$$
\begin{aligned}
d\left(x^{*}, T x^{*}\right) & \leq d\left(x^{*}, T^{2} x^{*}\right)+d\left(T^{2} x^{*}, T x^{*}\right) \\
& \leq \frac{r}{2-r} \delta\left(T x^{*}, x^{*}\right)+\frac{r^{2}}{2(2-r)} d\left(x^{*}, T x^{*}\right) .
\end{aligned}
$$

Similarly, we have

$$
\begin{aligned}
\delta\left(x^{*}, T x^{*}\right) & \leq \delta\left(x^{*}, T^{2} x^{*}\right)+\delta\left(T^{2} x^{*}, T x^{*}\right) \\
& \leq \frac{r}{2-r} d\left(T x^{*}, x^{*}\right)+\frac{r^{2}}{2(2-r)} \delta\left(x^{*}, T x^{*}\right) .
\end{aligned}
$$

If $\delta\left(x^{*}, T x^{*}\right) \leq d\left(x^{*}, T x^{*}\right)$, then (10) implies

$$
d\left(T x^{*}, x^{*}\right) \leq \frac{r}{2-r}\left(1+\frac{r}{2}\right) d\left(x^{*}, T x^{*}\right) .
$$

Since $\frac{r}{2-r}\left(1+\frac{r}{2}\right)<1$, for all $r \in\left[0, r_{0}\left[\right.\right.$, we obtain $T x^{*}=x^{*}$.

If $d\left(x^{*}, T x^{*}\right) \leq \delta\left(x^{*}, T x^{*}\right),(11)$ implies

$$
\delta\left(T x^{*}, x^{*}\right) \leq \frac{r}{2-r}\left(1+\frac{r}{2}\right) \delta\left(x^{*}, T x^{*}\right),
$$

which gives $T x^{*}=x^{*}$. These conclusions contradict the fact $T x^{*} \neq x^{*}$, if $r \in\left[0, r_{0}[\right.$.

Now assume $r \in\left[r_{0}, 1\right.$ [ and let $z \in A \backslash\left\{x^{*}\right\}$ where $A=\left\{z \in X / z\right.$ and $x_{n}$ are comparable, $\forall n \in \mathbb{N}\}$. For large integers, we have $\psi_{r_{0}}(r) d\left(T x_{n}, x_{n}\right) \leq d\left(x_{n}, z\right)$ and $x_{n}$ is comparable to $z$ for all $n$.

Thus

$$
\left\{\begin{array}{l}
d\left(T x_{n}, T z\right) \leq r \max \left\{\frac{d\left(x_{n}, T z\right)+\delta\left(z, T x_{n}\right)}{2}, d\left(x_{n}, T x_{n}\right)\right\} \\
\left.\delta\left(T x_{n}, T z\right) \leq r \max \left\{\frac{\delta\left(x_{n}, T z\right)+d\left(z, T x_{n}\right)}{2}, \delta\left(x_{n}, T x_{n}\right)\right\}\right\}
\end{array}\right.
$$


In the limit case, we obtain

$$
\left\{\begin{array}{l}
d\left(x^{*}, T z\right) \leq r \frac{d\left(x^{*}, T z\right)+\delta\left(z, x^{*}\right)}{2}, \\
\delta\left(x^{*}, T z\right) \leq r \frac{\delta\left(x^{*}, T z\right)+d\left(z, x^{*}\right)}{2},
\end{array}\right.
$$

and consequently

$$
\left\{\begin{array}{l}
d\left(x^{*}, T z\right) \leq \frac{r}{2-r} \delta\left(z, x^{*}\right), \\
\delta\left(x^{*}, T z\right) \leq \frac{r}{2-r} d\left(z, x^{*}\right) .
\end{array}\right.
$$

If $\delta\left(z, x^{*}\right) \leq d\left(z, x^{*}\right)$, we have

$$
d(z, T z) \leq d\left(z, x^{*}\right)+d\left(x^{*}, T z\right) \leq \frac{1}{1-\frac{r}{2}} d\left(z, x^{*}\right) \leq \frac{1}{1-r} d\left(z, x^{*}\right)
$$

and if $d\left(z, x^{*}\right) \leq \delta\left(z, x^{*}\right)$, we have

$$
\delta(z, T z) \leq \delta\left(z, x^{*}\right)+\delta\left(x^{*}, T z\right) \leq \frac{1}{1-r} \delta\left(z, x^{*}\right) .
$$

Otherwise, one of the inequalities

$$
\psi_{r_{0}}(r) d(z, T z) \leq d\left(z, x^{*}\right) \quad \text { or } \quad \psi_{r_{0}}(r) \delta(z, T z) \leq \delta\left(z, x^{*}\right)
$$

is satisfied.

If $\left(x_{n}\right)_{n}$ is a stationary sequence, we have $x^{*}=T x^{*}$ and if not, there exists a subsequence $\left(x_{\phi(n)}\right)_{n}$, such that $x_{\phi(n)} \neq x^{*}$, for all $n$. And consequently, for $z=x_{\phi(n)}$, we have

$$
d\left(T x_{\phi(n)}, T x^{*}\right) \leq r \max \left\{\frac{d\left(x_{\phi(n)}, T x^{*}\right)+\delta\left(x^{*}, T x_{\phi(n)}\right)}{2}, d\left(x_{\phi(n)}, T x_{\phi(n)}\right)\right\},
$$

which leads to

$$
d\left(x^{*}, T x^{*}\right) \leq r \frac{d\left(x^{*}, T x^{*}\right)}{2} .
$$

These considerations permit us to conclude that $x^{*}=T x^{*}$. These conclusions contradict the fact $T x^{*} \neq x^{*}$.

And then we conclude that $T x^{*}=x^{*}$.

Proposition 3.7 Under the same conditions of Theorem 3.6, if for any pair $\{x, y\} \subset X$ admits an upper bound or a lower bound, then $T$ has a unique fixed point.

Proof Suppose that there exist two fixed points $x, y \in X$ of $T$, from the hypothesis there exists $z$ in $X$ such that $x \preceq z$ and $y \preceq z$.

By the monotony of $T$,

$$
x=T^{n}(x) \preceq T^{n}(z),
$$


for all $n \geq 0$. Set $z_{n}=T^{n}(z)$, since

$$
d(x, T x) \leq d\left(x, z_{n}\right),
$$

for all integer $n$, then

$$
\left\{\begin{array}{l}
d\left(x, z_{n+1}\right) \leq r \max \left\{\frac{d\left(x, z_{n+1}\right)+\delta\left(z_{n}, x\right)}{2}, d(x, x)\right\}, \\
\delta\left(x, z_{n+1}\right) \leq r \max \left\{\frac{\delta\left(x, z_{n+1}\right)+d\left(z_{n}, x\right)}{2}, \delta(x, x)\right\},
\end{array}\right.
$$

so

$$
\left\{\begin{array}{l}
d\left(x, z_{n+1}\right) \leq \frac{r}{2-r} \delta\left(x, z_{n}\right), \\
\delta\left(x, z_{n+1}\right) \leq \frac{r}{2-r} d\left(x, z_{n}\right),
\end{array}\right.
$$

for all $n \geq 0$. Therefore,

$$
\begin{aligned}
d\left(x, z_{n}\right) & \leq\left(\frac{r}{2-r}\right)^{2} d\left(z_{n-2}, x\right) \\
& \leq\left(\frac{r}{2-r}\right)^{3} d\left(z_{n-3}, x\right) \\
& \vdots \\
& \leq\left(\frac{r}{2-r}\right)^{n} D_{n, n}(z, x),
\end{aligned}
$$

where

$$
D_{n, k}(z, x)= \begin{cases}d\left(z_{n-k}, x\right) & \text { if } k \text { is even and } k \in \mathbb{N}^{*} \\ \delta\left(z_{n-k}, x\right) & \text { if } k \text { is odd and } k \in \mathbb{N}^{*}\end{cases}
$$

Hence,

$$
\lim _{n \rightarrow+\infty} d\left(z_{n}, x\right)=0 .
$$

In the same way we prove that

$$
\lim _{n \rightarrow+\infty} d\left(z_{n}, y\right)=0 .
$$

By the triangle inequality,

$$
d(x, y) \leq d\left(x, z_{n}\right)+d\left(y, z_{n}\right) \quad \forall n \in \mathbb{N}
$$

and by (12) and (13) we conclude that $x=y$.

Corollary 3.8 Let $(X, d, \preceq)$ be an ordered metric space such that $(X, d)$ is complete, and $T$ a nondecreasing mapping on $X$ and $r_{0} \in[0,2(\sqrt{2}-1)[$. Assume that there exists $r \in[0,1$ [ 
such that, for all $(x, y) \in X^{2}, \psi_{r_{0}}(r) d(x, T x) \leq d(x, y)$ implies

$$
d(T x, T y) \leq r \max \left\{\frac{d(x, T y)+d(y, T x)}{2}, d(x, T x)\right\} .
$$

If there exists $x_{0} \in X$ such that $x_{0} \preceq T x_{0}$ and $X$ satisfies the property $(P)$ for $d$, then there exists an element $x^{*} \in X$ such that $T x^{*}=x^{*}$ and $\lim _{n \rightarrow+\infty} T^{n} x_{0}=x^{*}$. Moreover, the fixed point of $T$ is unique iffor all any pair $\{x, y\} \subset X$ admits an upper bound or a lower bound.

Theorem 3.9 Let $(X, \preceq)$ be a partially ordered set endowed with two metrics $d$ and $\delta$ such that $(X, d)$ is complete. Let $T$ be a monotone nondecreasing mapping; and assume that there exists $r \in\left[0, \frac{1}{2}\right.$ [ such that, for all comparable $x, y \in X$, one of the following conditions:

(i) $d(x, T x) \leq d(x, y)$,

(ii) $\delta(x, T x) \leq \delta(x, y)$,

implies

$$
\left\{\begin{array}{l}
d(T x, T y) \leq r \max \{d(x, T y), \delta(y, T x)\} \\
\delta(T x, T y) \leq r \max \{\delta(x, T y), d(y, T x)\}
\end{array}\right.
$$

If there exists $x_{0} \in X$ such that $x_{0} \preceq T x_{0}$ and $X$ satisfies the property $(P)$ for $d$, then there exists $x^{*} \in X$ such that $T x^{*}=x^{*}$ and

$$
\lim _{n \rightarrow+\infty} d\left(T^{n} x_{0}, x^{*}\right)=\lim _{n \rightarrow+\infty} \delta\left(T^{n} x_{0}, x^{*}\right)=0
$$

Moreover, the fixed point of $T$ is unique iffor all any pair $\{x, y\} \subset X$ admits an upper bound or a lower bound.

Proof Let $x_{0} \in X$, if $T\left(x_{0}\right)=x_{0}$, then the proof is finished. Suppose that

$$
T\left(x_{0}\right) \neq x_{0} .
$$

First step. We define a sequence $\left(x_{n}\right)_{n}$ by $x_{n}=T^{n} x_{0}$, for $n \geq 0$. Following the same lines of the proof of Theorem 3.1, the sequence $\left(x_{n}\right)_{n}$ is monotone for the partial order $\preceq$.

We have $d\left(x_{n}, T x_{n}\right) \leq d\left(x_{n}, x_{n+1}\right)$, then

$$
\left\{\begin{array}{l}
d\left(T x_{n}, T x_{n+1}\right) \leq r \max \left\{d\left(x_{n}, T x_{n+1}\right), \delta\left(x_{n+1}, T x_{n}\right)\right\} \\
\delta\left(T x_{n}, T x_{n+1}\right) \leq r \max \left\{\delta\left(x_{n}, T x_{n+1}\right), d\left(x_{n+1}, T x_{n}\right)\right\}
\end{array}\right.
$$

so

$$
\left\{\begin{array}{l}
d\left(T x_{n}, T x_{n+1}\right) \leq r d\left(x_{n}, x_{n+2}\right) \\
\delta\left(T x_{n}, T x_{n+1}\right) \leq r \delta\left(x_{n}, x_{n+2}\right)
\end{array}\right.
$$

and then

$$
\left\{\begin{array}{l}
d\left(x_{n+1}, x_{n+2}\right) \leq \frac{r}{1-r} d\left(x_{n}, x_{n+1}\right), \\
\delta\left(x_{n+1}, x_{n+2}\right) \leq \frac{r}{1-r} \delta\left(x_{n}, x_{n+1}\right) .
\end{array}\right.
$$


We have $\frac{r}{1-r} \in\left[0,1\left[,\left(x_{n}\right)_{n \geq 0}\right.\right.$ is a Cauchy sequence for $d$ and $\delta$ Then there exists $x^{*}$ in $X$ such that $\lim _{n} d\left(x_{n}, x^{*}\right)=0$.

Second step. Assume that $\left(\delta\left(x_{n}, x^{*}\right)\right)_{n}$ does not converge to 0 . Then

$$
\delta\left(x_{n}, T x_{n}\right) \leq \delta\left(x_{n}, x^{*}\right), \quad \text { for large integers } n
$$

since $x_{n} \preceq x^{*}$ for all $\mathrm{n}$, which leads to

$$
\left\{\begin{array}{l}
d\left(T x_{n}, T x^{*}\right) \leq r \max \left\{d\left(x_{n}, T x^{*}\right), \delta\left(x^{*}, T x_{n}\right)\right\}, \\
\delta\left(T x_{n}, T x^{*}\right) \leq r \max \left\{\delta\left(x_{n}, T x^{*}\right), d\left(x^{*}, T x_{n}\right)\right\} .
\end{array}\right.
$$

The second equation in (14) gives

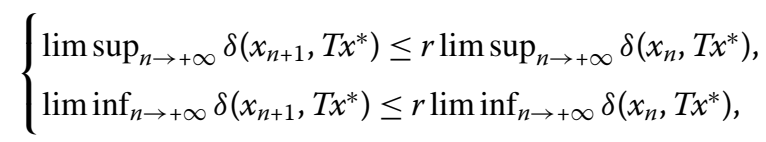

we obtain $\lim \sup _{n \rightarrow+\infty} \delta\left(x_{n}, T x^{*}\right)=\liminf _{n \rightarrow+\infty} \delta\left(x_{n}, T x^{*}\right)=0$; then $\lim _{n \rightarrow+\infty} \delta\left(x_{n}, T x^{*}\right)=0$ and $T x^{*} \neq x^{*}$; hence $d\left(x_{n}, T x_{n}\right) \leq d\left(x_{n}, T x^{*}\right)$, for large integers. Since $x_{n} \preceq T x^{*}$ for all $\mathrm{n}$, it follows that

$$
d\left(x_{n+1}, T^{2} x^{*}\right) \leq r \max \left\{d\left(x_{n}, T^{2} x^{*}\right), \delta\left(x_{n+1}, T x^{*}\right)\right\}
$$

and then $T^{2} x^{*}=x^{*}$.

We put $y=T x^{*}$. From the property $(\mathrm{P}) x^{*} \preceq T x^{*}$, and $\delta\left(x^{*}, T x^{*}\right) \leq \delta\left(x^{*}, y\right)$ we have

$$
d\left(T x^{*}, T^{2} x^{*}\right) \leq r d\left(x^{*}, T^{2} x^{*}\right) .
$$

Consequently $T x^{*}=x^{*}$. This contradiction shows that $\lim _{n} \delta\left(x_{n}, x^{*}\right)=0$.

Third step. Assume $T x^{*} \neq x^{*}$. As above, we have

$$
d\left(x_{n}, T x_{n}\right) \leq d\left(x_{n}, T x^{*}\right), \quad \text { for large integers } n,
$$

and then

$$
\left\{\begin{array}{l}
d\left(x_{n+1}, T^{2} x^{*}\right) \leq r \max \left\{d\left(x_{n}, T^{2} x^{*}\right), \delta\left(x_{n+1}, T x^{*}\right)\right\}, \\
\delta\left(x_{n+1}, T^{2} x^{*}\right) \leq r \max \left\{\delta\left(x_{n}, T^{2} x^{*}\right), d\left(x_{n+1}, T x^{*}\right)\right\} .
\end{array}\right.
$$

If $n$ goes to infinity, we obtain

$$
\left\{\begin{array}{l}
d\left(x^{*}, T^{2} x^{*}\right) \leq r \delta\left(x^{*}, T x^{*}\right) \\
\delta\left(x^{*}, T^{2} x^{*}\right) \leq r d\left(x^{*}, T x^{*}\right)
\end{array}\right.
$$

For $y=T x^{*}$, we have $\delta\left(x^{*}, T x^{*}\right) \leq \delta\left(x^{*}, y\right)$, so

$$
\left\{\begin{array}{l}
d\left(T x^{*}, T^{2} x^{*}\right) \leq r d\left(x^{*}, T^{2} x^{*}\right), \\
\delta\left(T x^{*}, T^{2} x^{*}\right) \leq r \delta\left(x^{*}, T^{2} x^{*}\right) .
\end{array}\right.
$$


Assume that $\delta\left(x^{*}, T x^{*}\right) \leq d\left(x^{*}, T x^{*}\right)$, we have

$$
d\left(x^{*}, T x^{*}\right) \leq d\left(x^{*}, T^{2} x^{*}\right)+d\left(T^{2} x^{*}, T x^{*}\right)
$$

with

$$
\left\{\begin{array}{l}
d\left(x^{*}, T^{2} x^{*}\right) \leq r \delta\left(x^{*}, T x^{*}\right) \leq r d\left(x^{*}, T x^{*}\right), \\
d\left(T^{2} x^{*}, T x^{*}\right) \leq r d\left(x^{*}, T^{2} x^{*}\right) \leq r^{2} d\left(x^{*}, T x^{*}\right) .
\end{array}\right.
$$

Then

$$
d\left(x^{*}, T x^{*}\right) \leq d\left(x^{*}, T^{2} x^{*}\right)+d\left(T^{2} x^{*}, T x^{*}\right) \leq\left(r+r^{2}\right) d\left(x^{*}, T x^{*}\right) .
$$

Since $r^{2}+r<1$, we obtain $d\left(x^{*}, T x^{*}\right)=0$.

If $\delta\left(x^{*}, T x^{*}\right) \geq d\left(x^{*}, T x^{*}\right)$; we permute $d$ and $\delta$, and we obtain $\delta\left(x^{*}, T x^{*}\right)=0$. These conclusions contradict the fact $T x^{*} \neq x^{*}$.

And then we conclude that $T x^{*}=x^{*}$.

For the uniqueness, we suppose that there exist two fixed points $x, y \in X$, from the hypothesis, there exists $z$ in $X$ such that $x \preceq z$ and $y \preceq z$.

By the monotony of $T$,

$$
x=T^{n}(x) \preceq T^{n}(z),
$$

for all $n \geq 0$. Set $z_{n}=T^{n}(z)$, since

$$
d(x, T x) \leq d\left(x, z_{n}\right),
$$

for all integer $n$, we have

$$
\left\{\begin{array}{l}
d\left(x, z_{n+1}\right) \leq r \max \left\{d\left(x, z_{n+1}\right), \delta\left(z_{n}, x\right)\right\}, \\
\delta\left(x, z_{n+1}\right) \leq r \max \left\{\delta\left(x, z_{n+1}, d\left(z_{n}, x\right)\right\}\right.
\end{array}\right.
$$

so

$$
\left\{\begin{array}{l}
d\left(x, z_{n+1}\right) \leq r \delta\left(z_{n}, x\right) \\
\delta\left(x, z_{n+1}\right) \leq r d\left(z_{n}, x\right)
\end{array}\right.
$$

for all $n \geq 0$. Therefore,

$$
\begin{aligned}
d\left(x, z_{n}\right) & \leq r^{2} d\left(z_{n-2}, x\right) \\
& \leq r^{3} d\left(z_{n-3}, x\right) \\
& \vdots \\
& \leq r^{n} D_{n, n}(z, x),
\end{aligned}
$$


where

$$
D_{n, k}(z, x)= \begin{cases}d\left(z_{n-k}, x\right) & \text { if } k \text { is even and } 2 \leq k \leq n \\ \delta\left(z_{n-k}, x\right) & \text { if } k \text { is odd and } 2 \leq k \leq n\end{cases}
$$

Hence,

$$
\lim _{n \rightarrow+\infty} d\left(z_{n}, x\right)=0
$$

In the same way we prove that

$$
\lim _{n \rightarrow+\infty} d\left(z_{n}, y\right)=0
$$

By the triangle inequality,

$$
d(x, y) \leq d\left(x, z_{n}\right)+d\left(y, z_{n}\right) \quad \forall n \in \mathbb{N},
$$

and (16), (17) we conclude that $x=y$.

Example 3.10 We consider the space $X=[0,1]$ ordered by " $\preceq$ " which is the reverse order of the usual order between the reals ( $x \preceq y \Leftrightarrow x \geq y$ ) and endowed with the usual metric $d$ and the metric defined by

$$
\delta(x, y)= \begin{cases}x+y & \text { if } x \neq y \\ 0 & \text { if } x=y\end{cases}
$$

We define

$$
\begin{aligned}
T: \quad X & \longrightarrow X, \\
x & \longmapsto \frac{\sin \left(x^{3}\right)}{6} .
\end{aligned}
$$

Set $r=\frac{4}{9}$. The map $T$ is nondecreasing, $X$ satisfies the property (P) and $1 \preceq T(1)$.

For all $x, y \in X$ such that $x \preceq y$, we have $r>\frac{1}{6}$, then $\frac{6 r-1}{r-1} \geq 1$ and $\sin \left(y^{3}\right) \leq \frac{6 r-1}{r-1} x$, hence

$$
\begin{aligned}
d(T x, T y) & =\frac{\sin \left(x^{3}\right)-\sin \left(y^{3}\right)}{6} \leq \frac{x-\sin \left(y^{3}\right)}{6} \leq r\left(x-\frac{\sin \left(y^{3}\right)}{6}\right) \\
& \leq r \max \{d(x, T y), \delta(y, T x)\} .
\end{aligned}
$$

Since $(1-r) \sin \left(y^{3}\right) \leq(1-r) y \leq(6 r-1) x$,

$$
\delta(T x, T y)=\frac{\sin \left(x^{3}\right)+\sin \left(y^{3}\right)}{6} \leq \frac{x+\sin \left(y^{3}\right)}{6} \leq r\left(x+\frac{\sin \left(y^{3}\right)}{6}\right) \leq r \max \{\delta(x, T y), d(y, T x)\} .
$$

Hence, $T$ has a unique fixed point $x^{*}=0$. 
Corollary 3.11 Let $(X, d, \preceq)$ be an ordered metric space such that $(X, d)$ is complete and $T$ a nondecreasing mapping on $(X, \preceq)$. If there exists $r \in\left[0, \frac{1}{2}[\right.$ such that, for all comparable $x, y \in X$,

$$
d(x, T x) \leq d(x, y) \quad \text { implies } \quad d(T x, T y) \leq r \max \{d(x, T y), d(y, T x)\} .
$$

If there exists $x_{0} \in X$ such that $x_{0} \preceq T x_{0}$ and $X$ satisfies the property $(P)$ for $d$, then there exists an element $x^{*} \in X$ such that $T x^{*}=x^{*}$ and $\lim _{n \rightarrow+\infty} T^{n} x_{0}=x^{*}$. Moreover, the fixed point of $T$ is unique if for all any pair $\{x, y\} \subset X$ admits an upper bound or a lower bound.

\section{Application}

Motivated by [11], in this section we apply Theorem 3.9 to study the existence and uniqueness of solution for the following general nonlinear matrix equation:

$$
X^{q}-A^{*} F(X) A=Q, \quad q>2, X \in \mathcal{P}(n),
$$

where $Q$ is $n \times n$ positive define matrix, $\mathcal{P}(n)$ is the set of all $n \times n$ Hermitian positivedefinite matrices, $A$ is $n \times n$ nonsingular matrix, $A^{*}$ is the Hermitian transpose of the matrix $A$ and $F: \mathcal{E}(n) \rightarrow \mathcal{E}(n)$ is a self-adjoint operator such that $\mathcal{E}(n)$ is a nonempty subset of $\mathcal{P}(n)$. This type of matrix equation arises in control theory, ladder networks, dynamic programming, stochastic filtering and statistics, etc. For $M, N \in \mathcal{P}(n)$, the notation $M \prec N$ means that $N-M$ is positive definite. We equipped $\mathcal{P}(n)$ with the partial ordered " $\preceq$ " given by

$$
M \preceq N \quad \Longleftrightarrow \quad M=N \quad \text { or } \quad M \prec N .
$$

We denote by $\|\cdot\|$ the spectral norm, i.e., $\|A\|=\sqrt{\rho\left(A^{*} A\right)}=\left\|A^{*}\right\|$ where $\rho\left(A^{*} A\right)$ is the largest eigenvalue of $A^{*} A$.

Let $r \in \mathbb{N}^{*}$, the function

$$
\begin{aligned}
\delta_{r}: \quad \mathcal{P}(n) \times \mathcal{P}(n) \longrightarrow \mathbb{R}_{+}, \\
(A, B) \longmapsto\left\|A^{r}-B^{r}\right\|,
\end{aligned}
$$

is a metric on $\mathcal{P}(n)$. In fact, it is easy to show that $\delta$ satisfies:

(i) symmetry, that is, $\delta_{r}(A, B)=\delta_{r}(A, B), \forall A, B \in \mathcal{P}(n)$.

(ii) the triangle inequality, that is, $\delta_{r}(A, B) \leq \delta_{r}(A, Z)+\delta_{r}(Z, B), \forall A, B, Z \in \mathcal{P}(n)$.

Moreover, $\delta_{r}$ satisfies the identity of indiscernibles, i.e., $\delta_{r}(A, B)=0 \Longleftrightarrow A=B$. Indeed, let $A, B \in \mathcal{P}(n)$, we have

$$
\delta_{r}(A, B)=0 \quad \Longleftrightarrow \quad A^{r}=B^{r} .
$$

Let $\lambda>0$ be an eigenvalue of $A$ and $X$ a vector in the eigenspace $E_{\lambda}(A)$ associated with $\lambda$. We have

$$
\begin{aligned}
A \cdot X=\lambda X \quad & \Longrightarrow \quad A^{r} \cdot X=\lambda^{r} X \\
& \Longleftrightarrow \quad B^{r} \cdot X=\lambda^{r} X
\end{aligned}
$$




$$
\begin{aligned}
& \Longleftrightarrow \quad\left(\left(\frac{1}{\lambda} B\right)^{r}-I_{n}\right) \cdot X=0 \\
& \Longleftrightarrow \quad \prod_{k=1}^{r-1}\left(\frac{1}{\lambda} B-e^{\left(2 i \frac{k \pi}{r}\right)} I_{n}\right)\left(\frac{1}{\lambda} B-I_{n}\right) \cdot X=0 .
\end{aligned}
$$

Since all the eigenvalues of $B$ are strictly positive, $B-\lambda e^{2 i \frac{k \pi}{r}} I_{n}$ is an inversible matrix. Hence,

$$
\begin{aligned}
\left(\frac{1}{\lambda} B-I_{n}\right) \cdot X=0 & \Longleftrightarrow B \cdot X=\lambda X \\
& \Longleftrightarrow X \in E_{\lambda}(B) .
\end{aligned}
$$

Thus, $E_{\lambda}(A) \subset E_{\lambda}(B)$. In the same way we have $E_{\lambda}(B) \subset E_{\lambda}(A)$. Therefore,

$$
E_{\lambda}(A)=E_{\lambda}(B)
$$

and since $A$ and $B$ are diagonalizable, $A=B$.

In the sequel, we consider the space $\mathcal{P}(n)$ equipped by the metric $\delta_{q}$ and the Thompson metric $d: \mathcal{P}(n) \times \mathcal{P}(n) \longrightarrow \mathbb{R}_{+}$given by

$$
d(A, B)=\max \{\ln (W(A / B)), \ln (W(B / A))\}=\left\|\ln \left(A^{-\frac{1}{2}} B A^{-\frac{1}{2}}\right)\right\|
$$

where $W(A / B)=\inf \{\lambda>0: A \leq \lambda B\}=\lambda_{\max }\left(B^{-\frac{1}{2}} A B^{-\frac{1}{2}}\right)$. It is easy to verify that $(\mathcal{P}(n), d)$ is a complete metric space (see [14]).

In the following lemmas, we give some elegant properties of the Thompson metric which play an important role in the proof of our main result of this section.

Lemma 4.1 ([12]) Let $d: \mathcal{P}(n) \times \mathcal{P}(n) \longrightarrow \mathbb{R}_{+}$be a Thompson metric on the open convex cone $\mathcal{P}(n)$, then, for any $A, B \in \mathcal{P}(n)$ and nonsingular matrix $N$, we have the following conditions:

1. $d(A, B)=d\left(A^{-1}, B^{-1}\right)=d\left(N^{*} A N, N^{*} B N\right)$, where $A^{-1}, B^{-1}$ are the inversion of matrices $A$ and $B$, respectively;

2. $d\left(A^{r}, B^{r}\right) \leq r d(A, B), r \in[0,1]$;

3. $d\left(N^{*} A^{r} N, N^{*} B^{r} N\right) \leq|r| d(A, B), r \in[-1,1]$.

Lemma 4.2 ([12]) For any $A, B, C, D \in \mathcal{P}(n)$,

$$
d(A+C, B+D) \leq \max \{d(A, B), d(C, D)\} .
$$

Especially, $d(A+C, B+C) \leq d(A, B)$.

Lemma 4.3 ([21]) For any $A, B \in \mathcal{P}(n)$, if $A \preceq B$, then $A^{r} \preceq B^{r}$ for all $\left.\left.r \in\right] 0,1\right]$, and $B^{r} \preceq A^{r}$ for all $r \in[-1,0[$.

Theorem 4.4 Let $X_{0} \in \mathcal{P}(n)$ and $\mathcal{E}(n)=\left\{X \in \mathcal{P}(n): X_{0} \preceq X\right\}$. If the operator $F$ is nondecreasing and for all $X, Y \in \mathcal{E}(n)$ comparable, one of the assertions

(i) $\left\|\ln \left(X^{-\frac{1}{2}}\left(Q+A^{*} F(X) A\right)^{\frac{1}{q}} X^{-\frac{1}{2}}\right)\right\| \leq\left\|\ln \left(X^{-\frac{1}{2}} Y X^{-\frac{1}{2}}\right)\right\|$, 
(ii) $\left\|X^{q}-Q-A^{*} F(X) A\right\| \leq\left\|X^{q}-Y^{q}\right\|$,

implies the system

$$
\left\{\begin{array}{l}
\left.\left\|\ln \left(F(X)^{-\frac{1}{2}} F(Y) F(X)^{-\frac{1}{2}}\right)\right\| \leq \| Y^{q}-Q-A^{*} F(X) A\right) \|, \\
\|F(X)-F(Y)\| \leq \frac{1}{q \rho\left(A^{*} A\right)}\left\|\ln \left(Y^{-\frac{1}{2}}\left(Q+A^{*} F(X) A\right)^{\frac{1}{q}} Y^{-\frac{1}{2}}\right)\right\|,
\end{array}\right.
$$

and $X_{0}^{q} \preceq Q$, then the matrix equation (18) has a unique solution.

Proof $\mathcal{E}(n)$ is nonempty subset of $\mathcal{P}(n)$. We show that $\mathcal{E}(n)$ is closed for distance $d$. In fact, let $\left(Y_{k}\right)_{k}$ be a sequence of $\mathcal{E}(n)$ which converges to $Y \in \mathcal{P}(n)$. If the set $\left\{k \in \mathbb{N}: Y_{k}=X_{0}\right\}$ is infinite, there exists a nondecreasing function $\phi: \mathbb{N} \longrightarrow \mathbb{N}$ such that $Y_{\phi(k)}=X_{0}, \forall k \in \mathbb{N}$, then $Y=X_{0} \in \mathcal{E}(n)$. If not, $Y_{k} \neq X_{0}$, for large integers $k$, then

$$
Y_{k} \in \mathcal{P}(n)+X_{0} \quad \text { for large integers } k
$$

Since $\mathcal{P}(n)$ is closed for distance $d$, because $(\mathcal{P}(n), d)$ is a complete metric space, $\mathcal{P}(n)+X_{0}$ is closed. As $Y_{k} \rightarrow Y$, then $Y \in \mathcal{P}(n)+X_{0}$. That is to say, $Y \in \mathcal{E}(n)$.

First step. Let $T: \mathcal{E}(n) \longrightarrow \mathcal{E}(n)$ be a function given by

$$
T(X)=\left(Q+A^{*} F(X) A\right)^{\frac{1}{q}}, \quad X \in \mathcal{E}(n) .
$$

Since $F$ is nondecreasing, $T$ is well defined and conserves the partial ordering on $\mathcal{E}(n)$. Indeed, let $X \in \mathcal{E}(n)$, we have

$$
X_{0} \preceq X \quad \Longleftrightarrow \quad Q+A^{*} F\left(X_{0}\right) A \preceq Q+A^{*} F(X) A .
$$

As $X_{0}^{q} \preceq Q$ and $A^{*} F\left(X_{0}\right) A \in \mathcal{P}(n)$, then $X_{0}^{q} \preceq Q+A^{*} F\left(X_{0}\right) A$. By Lemma 4.3 and (20), we have

$$
X_{0} \preceq\left(Q+A^{*} F(X) A\right)^{\frac{1}{q}}=T(X) \quad \Longleftrightarrow \quad T(X) \in \mathcal{E}(n) .
$$

Let $B, C \in \mathcal{E}(n)$ such that $B \preceq C$, we have $(T(C))^{q}-(T(B))^{q}=A^{*}(F(C)-F(B)) A$, a positive semidefinite matrix, $(T(B))^{q} \preceq(T(C))^{q}$. Combining Lemma 4.3 and $0 \leq \frac{1}{q}<1$, then $T(B) \preceq$ $T(C)$.

Second step. From the hypothesis, we have $X_{0}^{q} \preceq Q$. Since $A^{*} F\left(X_{0}\right) A \in \mathcal{P}(n)$,

$$
\begin{aligned}
Q+A^{*} F\left(X_{0}\right) A-X_{0}^{q} \in \mathcal{P}(n) & \Longleftrightarrow \quad X_{0}^{q} \prec Q+A^{*} F\left(X_{0}\right) A \\
& \Longrightarrow \quad X_{0}^{q} \preceq Q+A^{*} F\left(X_{0}\right) A .
\end{aligned}
$$

Hence $X_{0} \preceq T\left(X_{0}\right)$.

Next, we show that $\mathcal{E}(n)$ satisfies the property (P) for $d$, given in Definition 2.6. In fact, for (i), let $\left(X_{k}\right)_{k}$ be a nondecreasing sequence of $\mathcal{E}(n)$ which converges to $X \in \mathcal{E}(n)$.

Fix $m \in \mathbb{N}$ arbitrary. For all $k>m$,

$$
X_{m} \prec X_{k} \quad \Longleftrightarrow \quad X_{k}-X_{m} \in \mathcal{P}(n) .
$$


Then $\mathcal{P}(n)+X_{m}$ is closed, since $\mathcal{P}(n)$ is closed for distance $d$. As $X_{k} \rightarrow X$, then

$$
X \in \mathcal{P}(n)+X_{m} \quad \Longleftrightarrow \quad X-X_{m} \in \mathcal{P}(n) .
$$

Thus

$$
\begin{aligned}
X-X_{m} \in \mathcal{P}(n) & \Longleftrightarrow X_{m} \prec X \\
& \Longrightarrow \quad X_{m} \preceq X, \quad \forall m \in \mathbb{N} .
\end{aligned}
$$

For (ii), let $\left(X_{k}\right)_{k} \subset \mathcal{E}(n)$ and $Y \in \mathcal{E}(n)$ such that $X_{k} \rightarrow X$ and $X_{k} \preceq Y$, for all integer $k$. Then $X \preceq Y$. Indeed, if $X_{k}=Y$ for an infinity of the integer $k$, then there exists a nondecreasing function $\psi: \mathbb{N} \longrightarrow \mathbb{N}$ such that $Y_{\psi(k)}=Y, \forall k \in \mathbb{N}$, so $X=Y$. If not, $X_{k} \neq Y$, for large integers $k$, then

$$
\begin{aligned}
X_{k} \prec Y \quad & \Longleftrightarrow Y \in \mathcal{P}(n)+X_{k} \\
& \Longleftrightarrow \quad \exists A_{k} \in \mathcal{P}(n) \text { such that } \quad Y=A_{k}+X_{k}, \quad \text { for large integers } k
\end{aligned}
$$

Since

$$
\begin{aligned}
d\left(Y, A_{k}+X\right) & =d\left(A_{k}+X_{k}, A_{k}+X\right) \\
& \leq d\left(X_{k}, X\right),
\end{aligned}
$$

for large integers $k$, then $d\left(Y, A_{k}+X\right) \rightarrow 0$. Since $A_{k}+X \in \mathcal{P}(n)+X$ and $A_{k}+X \rightarrow Y$. Thus, $Y \in \mathcal{P}(n)+X$, consequently $X \preceq Y$.

Third step. Let $X, Y$ be two comparable elements in $\mathcal{E}(n)$. If we assume that

$$
d(X, T(X)) \leq d(X, Y) \quad \text { or } \quad \delta(X, T(X)) \leq \delta(X, Y),
$$

then one of the following assertions is verified:

(i) $\left\|\ln \left(X^{-\frac{1}{2}}\left(Q+A^{*} F(X) A\right)^{\frac{1}{q}} X^{-\frac{1}{2}}\right)\right\| \leq\left\|\ln \left(X^{-\frac{1}{2}} Y X^{-\frac{1}{2}}\right)\right\|$,

(ii) $\left\|X^{q}-Q-A^{*} F(X) A\right\| \leq\left\|X^{q}-Y^{q}\right\|$,

which implies

$$
\left\{\begin{array}{l}
\left.\left\|\ln \left(F(X)^{-\frac{1}{2}} F(Y) F(X)^{-\frac{1}{2}}\right)\right\| \leq \| Y^{q}-Q-A^{*} F(X) A\right) \|, \\
\|F(X)-F(Y)\| \leq \frac{1}{q \rho\left(A^{*} A\right)}\left\|\ln \left(Y^{-\frac{1}{2}}\left(Q+A^{*} F(X) A\right)^{\frac{1}{q}} Y^{-\frac{1}{2}}\right)\right\| .
\end{array}\right.
$$

Using Lemmas 4.1 and 4.2, we have

$$
\begin{aligned}
\left\|\ln \left(F(X)^{-\frac{1}{2}} F(Y) F(X)^{-\frac{1}{2}}\right)\right\| & =d(F(X), F(Y)) \\
& =d\left(A^{*} F(X) A, A^{*} F(Y) A\right) \\
& \geq d\left(Q+A^{*} F(X) A, Q+A^{*} F(Y) A\right) \\
& \geq q d(T(X), T(Y)) .
\end{aligned}
$$


And

$$
\begin{aligned}
\left\|Y^{q}-Q-A^{*} F(X) A\right\| & =\left\|Y^{q}-T(X)^{q}\right\| \\
& =\delta_{q}(Y, T(X)) .
\end{aligned}
$$

Thus, by (21), (22) and (23)

$$
d(T(X), T(Y)) \leq \frac{1}{q} \delta_{q}(Y, T(X)) \leq \frac{1}{q} \max \left\{d(X, T(Y)), \delta_{q}(Y, T(X))\right\} .
$$

On the other hand, we get

$$
\begin{aligned}
\|A\|^{2}\|F(X)-F(Y)\| & =\left\|A^{*}\right\|\|F(X)-F(Y)\|\|A\| \\
& \geq\left\|A^{*} F(X) A-A^{*} F(Y) A\right\| \\
& =\left\|T(X)^{q}-T(Y)^{q}\right\| .
\end{aligned}
$$

Also

$$
\left\|\ln \left(Y^{-\frac{1}{2}}\left(Q+A^{*} F(X) A\right)^{\frac{1}{q}} Y^{-\frac{1}{2}}\right)\right\|=d(Y, T(X)),
$$

then, by (21), (24) and (25),

$$
\delta_{q}(T(X), T(Y)) \leq \frac{1}{q} d(Y, T(X)) \leq \frac{1}{q} \max \left\{d(Y, T(X)), \delta_{q}(X, T(Y))\right\} .
$$

Therefore, there exists $r=\frac{1}{q} \in\left[0, \frac{1}{2}[\right.$ for all comparable elements $X, Y \in \mathcal{E}(n)$, one of the conditions:

(i) $d(X, T(X)) \leq d(X, Y)$,

(ii) $\delta_{q}(X, T(X)) \leq \delta_{q}(X, Y)$,

implies

$$
\left\{\begin{array}{l}
d(T(X), T(Y)) \leq r \max \left\{d(X, T(Y)), \delta_{q}(Y, T(X))\right\} \\
\delta_{q}(T(X), T(Y)) \leq r \max \left\{\delta_{q}(X, T(Y)), d(Y, T(X))\right\}
\end{array}\right.
$$

Thus, according to Theorem 3.9, we can easily draw the conclusion that there exists $X^{*} \in$ $\mathcal{E}(n)$ such that $T\left(X^{*}\right)=X^{*}$.

According to the properties of $\mathcal{P}(n)$, we find that every pair $\{X, Y\} \subset \mathcal{E}(n)$ admits an upper bound or a lower bound. In fact, let $X, Y \in \mathcal{E}(n)$, then there exists $Z=X+Y \in \mathcal{E}(n)$ such that $X \preceq Z$ and $Y \preceq Z$. Hence $T$ has a unique fixed point.

Therefore, $X^{*}$ is the unique solution of the matrix equation (18).

Example 4.5 We consider the space $\mathcal{E}(n)=\left\{X \in \mathcal{P}(n): \frac{1}{2} I_{n} \preceq X\right\}$ equipped by the metric $\delta_{3}$ and the Thompson metric $d$ where $I_{n}$ is the identity matrix. Consider the following general nonlinear matrix equation:

$$
X^{3}-A^{*} F(X) A=Q, \quad X \in \mathcal{P}(n),
$$


where $Q=A=I_{n}$ and $F: \mathcal{E}(n) \rightarrow \mathcal{E}(n)$ is a mapping defined by

$$
F(X)= \begin{cases}\frac{3}{4} I_{n}, & \text { if } \frac{1}{2} I_{n} \prec X \\ \frac{7}{10} I_{n}, & \text { if } X=\frac{1}{2} I_{n} .\end{cases}
$$

(1) $F$ is not a monotone nonexpansive mapping for the metric $\delta_{3}$ and the Thompson metric $d$,

(2) $F$ is monotone satisfies condition (19) for all $X, Y \in \mathcal{E}(n)$ comparable.

Setting $X=\frac{8}{15} I_{n}, Y=\frac{1}{2} I_{n}$, we obtain

$$
d(F(X), F(Y))=\ln \left(\frac{15}{14}\right)>\ln \left(\frac{16}{15}\right)=d(X, Y)
$$

However, $F$ is not a nonexpansive mapping for the Thompson metric $d$. Setting $X=\frac{27}{50} I_{n}$, $Y=\frac{1}{2} I_{n}$, we obtain

$$
\delta_{3}(F(X), F(Y))=\frac{631}{8000}>\frac{2029}{62,500}=\delta_{3}(X, Y) .
$$

Then $F$ is not a nonexpansive mapping for the metric $\delta_{3}$. Now, we show that $F$ is monotone satisfies condition (19). In fact, let $X \in \mathcal{E}(n) \backslash\left\{\frac{1}{2} I_{n}\right\}$ and $Y=\frac{1}{2} I_{n}$ such that $Y \preceq X$, we have

$$
d(F(X), F(Y))=d\left(\frac{3}{4} I_{n}, \frac{7}{10} I_{n}\right)=\left\|\ln \left(\frac{14}{15} I_{n}\right)\right\|=\ln \left(\frac{15}{14}\right)
$$

and

$$
\delta_{3}\left(Y,\left(Q+A^{*} F(X) A\right)^{\frac{1}{3}}\right)=\left\|Y^{3}-I_{n}-\frac{3}{4} I_{n}\right\|=\left\|\frac{1}{8} I_{n}-\frac{7}{4} I_{n}\right\|=\frac{13}{8} .
$$

Therefore

$$
d(F(X), F(Y)) \leq \delta_{3}\left(Y,\left(Q+A^{*} F(X) A\right)^{\frac{1}{3}}\right) .
$$

In the same way, we have

$$
\|F(X)-F(Y)\|=\left\|\frac{3}{4} I_{n}-\frac{7}{10} I_{n}\right\|=\frac{1}{20}
$$

and

$$
d\left(Y,\left(Q+A^{*} F(X) A\right)^{\frac{1}{3}}\right)=d\left(\frac{1}{2} I_{n},\left(\frac{7}{4} I_{n}\right)^{\frac{1}{3}}\right)=\left\|\ln \left(2 \sqrt[3]{\frac{7}{4}} I_{n}\right)\right\|=\ln \left(2 \sqrt[3]{\frac{7}{4}}\right) .
$$

Then

$$
\|F(X)-F(Y)\| \leq \frac{1}{3} d\left(Y,\left(Q+A^{*} F(X) A\right)^{\frac{1}{3}}\right) .
$$


Since $F$ is monotone it satisfies (27) and (28) for all $X, Y \in \mathcal{E}(n)$ comparable and there exists $X_{0}=\frac{1}{2} I_{n}$ such that $\left(X_{0}\right)^{3} \preceq Q$, then all the conditions of Theorem 4.4 are satisfied. Consequently, the matrix equation (26) has a unique solution $X^{*}=\sqrt[3]{\frac{7}{4}} I_{n}$.

Funding

We have no funding for this article.

Availability of data and materials

Data sharing not applicable to this article as no datasets were generated or analysed during the current study.

\section{Competing interests}

The authors declare that they have no competing interests.

\section{Authors' contributions}

All authors contributed equally and significantly in writing this article. All authors read and approved the final manuscript.

\section{Author details}

'Laboratory of Algebra, Analysis and Applications (L3A), Faculty of Sciences Ben M'Sik, Hassan II University of Casablanca, Casablanca, Morocco. ${ }^{2}$ CRMEF, Rabat, Morocco.

\section{Publisher's Note}

Springer Nature remains neutral with regard to jurisdictional claims in published maps and institutional affiliations.

Received: 29 March 2019 Accepted: 6 May 2020 Published online: 01 June 2020

\section{References}

1. Aliouche, A., Fisher, B.: A related fixed point theorem for two pairs of mappings on two complete metric spacs. Hacet. J. Math. Stat. 34, 39-45 (2005)

http://www.hjms.hacettepe.edu.tr/uploads/596a9157-b6cf-4ca5-afe9-e9ef9357d7d4.pdf

2. Bose, R.K., Roychowdhury, M.K.: Fixed point theorems for some generalized contractive multivalued mappings and fuzzy mappings. Mat. Vesn. 63(1), 7-26 (2011) http://www.emis.de/journals/MV/111/mv11102.pdf

3. Chaira, K., Marhrani, E.: Some related fixed points theorems for a pair of mapping on two metric spaces. Int. J. Pure Appl. Math. 93(2), 191-200 (2014) https://ijpam.eu/contents/2014-93-2/4/4.pdf

4. Chaira, K., Marhrani, E.: Some related fixed points theorems of metric spaces. Bull. Math. Anal. Appl. 7(2), 46-55 (2015) http://www.bmathaa.org/repository/docs/BMAA7-2-5.pdf

5. Chatterjea, S.K.: Fixed-point theorems. C. R. Acad. Bulg. Sci. 25, 727-790 (1972)

6. Doric, D., Lazovic, R.: Some Suzuki-type fixed point theorems for generalized multivalued mappings and applications. Fixed Point Theory Appl. 2011, Article ID 40 (2011). https://doi.org/10.1186/1687-1812-2011-40

7. Fisher, B.: Fixed point on two metric spaces. Glas. Mat. 16(36), 333-337 (1981)

8. Kannan, R.: Some results on fixed points. Bull. Calcutta Math. Soc. 60, 71-76 (1968)

9. Kannan, R.: Some results on fixed points II. Am. Math. Mon. 76, 405-408 (1969). https://doi.org/10.1080/00029890.1969.12000228

10. Kikkawa, M., Suzuki, T.: Three fixed point theorems for generalized contractions with constants in complete metric spaces. Nonlinear Anal., Theory Methods Appl. 69(9), 2942-2949 (2008). https://doi.org/10.1016/j.na.2007.08.064

11. Liao, A.P., Yao, G.Z., Duan, X.F.: Thompson metric method for solving a class of nonlinear matrix equation. Appl. Math. Comput. 216, 1831-1836 (2010). https://doi.org/10.1016/j.amc.2009.12.022

12. Lim, $Y .:$ Solving the nonlinear matrix equation $X=Q+\sum_{i}^{m} M_{i}^{*} X^{\delta_{i}} M_{i}$ via a contraction principle. Linear Algebra Appl. 430(4), 1380-1383 (2009). https://doi.org/10.1016/j.laa.2008.10.034

13. Nieto, J.J., Rodriguez-Lopez, R.: Contractive mapping theorems in partially ordered sets and applications to ordinary differential equations. Order 22(3), 223-239 (2005). https://doi.org/10.1007/s11083-005-9018-5

14. Nussbaum, R.D.: Hilbert's projective metric and iterated nonlinear maps. Mem. Am. Math. Soc. 391 (1988) https://doi.org/10.1090/memo/0391

15. Petrusel, A., Mot, G.: Fixed point theory for a new type of contractive multivalued operators. Nonlinear Anal., Theory Methods Appl. 70(9), 3371-3377 (2009). https://doi.org/10.1016/j.na.2008.05.005

16. Petrusel, A., Rus, I.A.: Fixed point theory in terms of a metric and of an order relation. Fixed Point Theory 20(2), 601-622 (2019). https://doi.org/10.24193/fpt-ro.2019.2.40

17. Ran, A.C.M., Reurings, M.C.B.: A fixed point theorem in partially ordered sets and some applications to matrix equations. Proc. Am. Math. Soc. 132(5), 1435-1443 (2004). https://doi.org/10.1090/S0002-9939-03-07220-4

18. Roman, S.: Lattices and Ordered Sets. Springer, Berlin (2008). ISBN 978-0-387-78901-9. https://doi.org/10.1007/978-0-387-78901-9

19. Singh, S.L., Mishra, S.N., Chugh, R., Kamal, R.: General common fixed point theorems and applications. J. Appl. Math. 2012, Article ID 902312 (2012). https://doi.org/10.1155/2012/902312

20. Suzuki, T:: A generalized Banach contraction principle that characterises metric completeness. Proc. Am. Math. Soc 136(5), 1861-1869 (2008). https://doi.org/10.1090/S0002-9939-07-09055-7

21. Zhan, X.: Computing the extremal positive definite solutions of a matrix equation. SIAM J. Sci. Comput. 17(5), 1167-1174 (1996). https://doi.org/10.1137/S1064827594277041 